\title{
Spectral Transmittance Characteristics of Holmium Oxide in Perchloric Acid Solution
}

\author{
Victor R. Weidner, Radu Mavrodineanu, Klaus D. Mielenz, Rance A. Velapoldi, \\ Kenneth L. Eckerle, and Bradley Adams \\ National Bureau of Standards, Gaithersburg, MD 20899
}

Accepted: November 28, 1984

\begin{abstract}
The work describes the methods and procedures used to determine the wavelengths of minimum transmittance of holmium oxide in perchloric acid solution. Measurements of spectral transmittance of the solutions were made by means of a high precision spectrophotometer over the wavelength range $200 \mathrm{~nm}$ to $680 \mathrm{~nm}$. The wavelength scale accuracy of this instrument was verified by extensive measurements of mercury and deuterium emission lines. The measurements of spectral transmittance of the holmium oxide solutions were made as a function of temperature, purity, concentration, and spectral bandwidth. Analysis of the uncertainties associated with these parameters and the uncertainties associated with the calibration of the instrument wavelength scale and the data analysis have resulted in an estimated uncertainty of $\pm 0.1 \mathrm{~nm}$ for the determination of the wavelengths of minimum transmittance of the holmium oxide solution.
\end{abstract}

Key words: holmium oxide; spectral bandwidth; spectrophotometer calibration; spectral transmittance; wavelength calibration; wavelength standard.

\section{Introduction}

This work describes the methods and procedures used to determine the wavelengths of minimum spectral transmittance of holmium oxide $\left(\mathrm{Ho}_{2} \mathrm{O}_{3}\right)$ in perchloric acid $\left(\mathrm{HClO}_{4}\right)$ solution in the spectral region 200 to 680 $\mathrm{nm}$. The object of this activity was to develop a standard for verifying the wavelength scale of uv/visible spectrophotometers, and to provide assistance toward improving the accuracy of measurements in the fields of

About the Authors: Victor R. Weidner is with NBS' Radiometric Physics Division, headed by Klaus D. Mielenz and served also by Kenneth L. Eckerle; Radu Mavrodineanu is associated with the Bureau's Inorganic Analytical Research Division as a reemployed annuitant; Rance A. Velapoldi is deputy director of the NBS Center for Analytical Chemistry; and Bradley Adams served the Bureau as a student worker. molecular absorption spectrometry or spectrophotometry $[1]^{1}$.

Holmium oxide in a glass matrix has been issued by the National Bureau of Standards (NBS) as a wavelength standard for the ultraviolet and visible spectrum since 1961 [2]. Didymium glass wavelength standards have been issued since 1945 . The didymium glass wavelength standards are available from NBS as Standard Reference Material 2009; 2010; 2013; and 2014 [3].

\section{Experimental \\ 2.1 Instrumentation}

Measurements of spectral transmittance of the holmium oxide solutions were made by means of a Varian Cary Model 2390 recording spectrophotometer ${ }^{2}$.

\footnotetext{
'Numbers in brackets indicate literature references.

${ }^{2}$ Certain commercial equipment or products are mentioned in this paper in order to adequately document the work. In no case does this imply that the equipment or product is being endorsed by NBS or that it is necessarily the best equipment or product for the application.
} 
The optical system of this double beam instrument consists of a filter predisperser and a double-pass double-sided grating monochromator. A deuterium lamp is used over the wavelength range 185 to $340 \mathrm{~nm}$ and a tungsten-halogen lamp is used over the wavelength range 340 to $3150 \mathrm{~nm}$ as a source. The spectral bandwidths are selectable from $0.07 \mathrm{~nm}$ to $3.6 \mathrm{~nm}$. Data can be recorded by a digital readout system with thermal printer and an analog chart display.

\subsection{Materials}

\subsubsection{Holmium Oxide}

The holmium oxide solutions were prepared by dissolving the powder in a $10 \%$ perchloric acid in distilled water. The purity of the holmium oxide specimens used in the preparation of these solutions was indicated by the manufacturer [4] to be $99.99 \%$ (Lot No. Ho-0-4-007) and $99.999 \%$ (Lot No. Ho-0-5-007). Solutions were prepared with $2 \%, 4 \%$, and $6 \%$ holmium oxide. These solutions were placed in $10 \mathrm{~mm}$ pathlength nonfluorescent fused silica cuvettes.

The actual form in which $\mathrm{Ho}$ is formed when $\mathrm{Ho}_{2} \mathrm{O}_{3}$ is dissolved in $\mathrm{HClO}_{4}$ is that of an aquo ion of the general formula $\mathrm{Ho}\left(\mathrm{H}_{2} \mathrm{O}\right)_{n}^{3}+$. The term "holmium oxide" and the spectral transmittances reported in the manuscript refer to this chemical species. Aqueous solutions of perchloric acid are used in this study to dissolve $\mathrm{Ho}_{2} \mathrm{O}_{3}$ since the resulting aquo ion is least likely to form complexes when subjected to changes in temperature and concentration [5].

The holmium oxide powder is stated by the manufacturer to have an average particle size of about $2 \mathrm{mi}-$ crometers. The perchloric acid was a nominal $70-72 \%$ reagent grade (considered here as $100 \%$ ). The distilled water was produced by thermal distillation. The aqueous solutions of holmium oxide in $10 \%$ perchloric acid were prepared by weighing 2,4 , or $6 \mathrm{~g}$ of the oxide and adding $10 \mathrm{~mL}$ of distilled water and $10 \mathrm{~mL}$ of perchloric acid. The holmium oxide was dissolved by heating at about $80^{\circ} \mathrm{C}$ for one hour. The clear solution was transferred quantitatively to a $100 \mathrm{~mL}$ volumetric flask and was brought to volume with distilled water at room temperature.

\subsubsection{Cells}

The cells used for the transmission measurements of the holmium oxide solutions were conventional nonfluorescent, fused silica, cuvettes with a nominal pathlength of $10 \mathrm{~mm}$. These cuvettes were provided with graded quartz-to-pyrex tubes with rubber caps.

\subsection{Measurement Techniques}

\subsubsection{Calibration of the Spectrophotometer Wavelength Scale}

The spectophotometer wavelength scale error was evaluated by measuring the emission spectrum of the instrument's deuterium lamp and the emission spectrum of a mercury pen lamp $[6,7,8]$.

The wavelength calibration was performed at the beginning of the holmium oxide solution measurements and again at the completion of the measurements. A number of emission lines of mercury and two emission lines of deuterium were used over the wavelength range $230 \mathrm{~nm}$ to $690 \mathrm{~nm}$ (see fig. 1). The wavelength scale errors were determined for spectral bandwidths of 0.1 $\mathrm{nm}, 1 \mathrm{~nm}, 2 \mathrm{~nm}$, and $3 \mathrm{~nm}$.

Each emission line was scanned at a rate of $0.01 \mathrm{~nm}$ per second and recorded on a scale of $0.2 \mathrm{~nm}$ per centi-

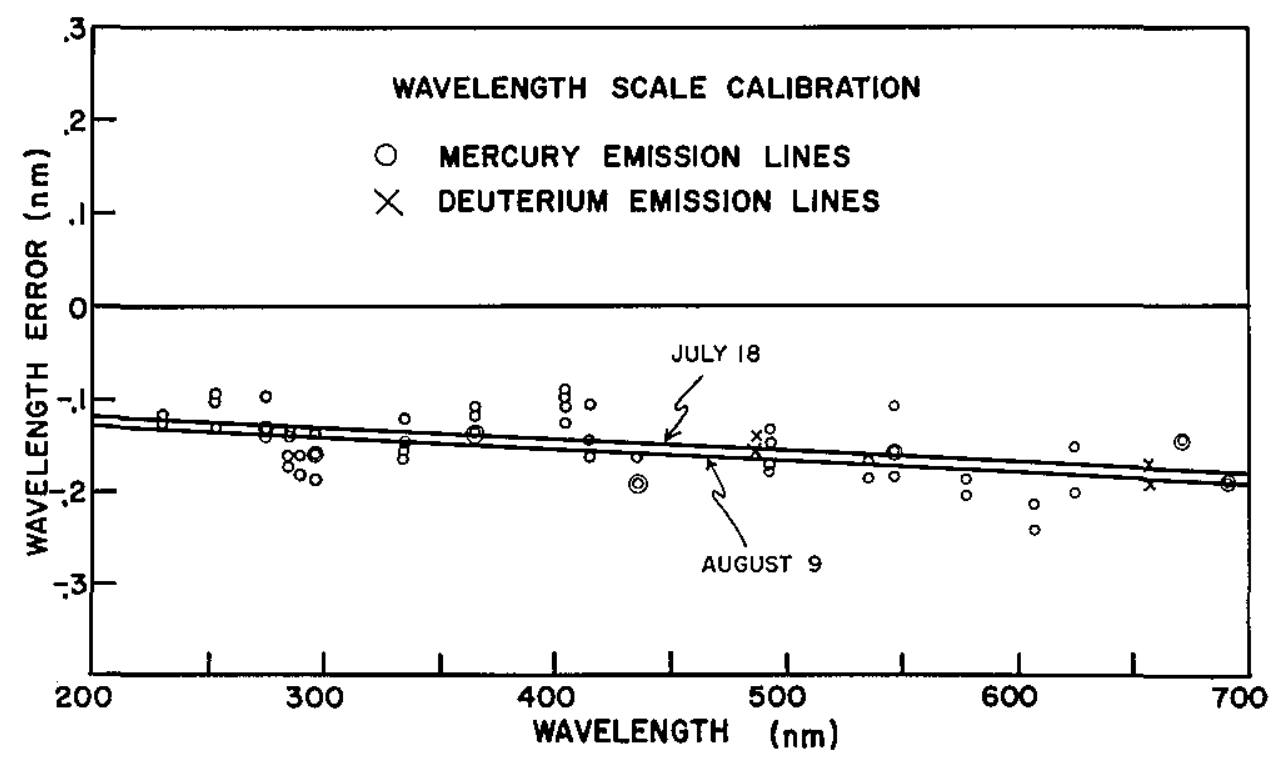

Figure 1-Wavelength scale calibration of the spectrophotometer. 
meter. The recorded emission peaks were bisected, using a proportional divider, to determine the wavelengths at the center of the recorded triangular lines. Using this technique, the wavelength scale was read to the nearest $0.01 \mathrm{~nm}$ with a precision of $\pm 0.005 \mathrm{~nm}$. The differences between the measured wavelengths of mercury emission lines and the true wavelengths are plotted in figure 1. A linear least squares fit of these wavelength differences for the two dates July 18 and August 9 (also shown in fig. 1) was used to correct the measured values of the holmium oxide transmission minima for errors in the wavelength scale of the instrument.

The wavelength error of the instrument was essentially the same for spectral bandwidths less than $1 \mathrm{~nm}$. However, at spectral bandwidths of $2 \mathrm{~nm}$ and $3 \mathrm{~nm}$, the wavelength error was slightly different and a different correction was required.

The deuterium lamp emission lines were measured at two wavelengths each day during the holmium oxide solution characterization to verify the accuracy of the wavelength scale. These measurements showed that the calibration of the wavelength scale is constant to \pm 0.02 $\mathrm{nm}$ after a nominal warm-up of one hour. These results, as well as the repeatability of the calibration with the mercury line source, indicate that the overall stability of the instrument wavelength scale was better than \pm 0.05 $\mathrm{nm}$ during the period of time required to complete the wavelength characterization of the holmium oxide solution.

The mercury pen lamp is mounted in the lamp positioning device that ordinarily holds the deuterium lamp of the spectrophotometer. The arc tube of the mercury pen was aligned parallel to the entrance slit of the monochromator. The lamp holder is equipped with screw adjustments for vertical and horizontal alignment of the source. The entrance slit cannot be directly observed. Therefore, the source was adjusted with instrument operating in the single-beam mode until a maximum signal is observed. To test the effect of the positioning of the mercury line source on the observed emission line maxima, the pen lamp was moved horizontally across the field of view of the entrance slit to the monochromator in approximately $0.22 \mathrm{~mm}$ steps for a total of 12 steps or a $2.64 \mathrm{~mm}$ distance. The results of this experiment showed that the recorded emission maxima varied by less than $\pm 0.01 \mathrm{~nm}$ for lamp positions within $\pm 1 \mathrm{~mm}$ of the center position. The center position corresponded to the position of maximum signal.

\subsubsection{Confirmation of Spectral Bandwidths}

The mercury line source was used to confirm the spectral bandwidth settings of the spectrophotometer. The mercury emission line at $435.8 \mathrm{~nm}$ was scanned for spectral bandwidths of $0.1 \mathrm{~nm}, 0.25 \mathrm{~nm}, 0.5 \mathrm{~nm}, 1 \mathrm{~nm}, 2$ $\mathrm{nm}$ and $3 \mathrm{~nm}$. The emission peak was normalized to $100 \%$ on the chart recorder by adjusting the instrument gain. The bandwidth at half peak height is approximately equal to the spectral bandwidth. The natural bandwidth of the emission line is much less than the instrument bandwidth. The recorded curve has a triangular symmetry for all settings of the monochromator slitwidths. For the above-mentioned nominal spectral bandwidth settings of the spectrophotometer, the measured spectral bandwidths were $0.092 \mathrm{~nm}, 0.228 \mathrm{~nm}$, $0.468 \mathrm{~nm}, 1.04 \mathrm{~nm}, 2.18 \mathrm{~nm}$, and $3.20 \mathrm{~nm}$, respectively. This technique for determining spectral bandwidths has some uncertainties due to assumptions made. However, it serves to confirm that the desired spectral bandwidths are closely approximated when the instrument is programmed to provide those settings; hence the effect on the transmittance minima is negligible.

\subsubsection{Determination of the Wavelengths of Minimum Transmittance}

The spectral transmittance of holmium oxide in an aqueous solution of perchloric acid exhibits many absorption bands in the ultraviolet and visible spectrum. The spectrum is shown in figure 2 for a $0.1 \mathrm{~nm}$ spectral bandwidth scan. The total number of observed absorption bands varies as a function of the spectral bandwidth used during the recording of the spectrum. There are approximately 14 major absorption features or bands between 200 and $650 \mathrm{~nm}$ that can be observed clearly for a wide range of spectral bandwidth settings. Most of the major absorption bands have lesser bands between them or in close association. These smaller bands are better resolved at bandwidths less than $1 \mathrm{~nm}$. Only the major absorption bands that could be of use as possible wavelength standards were selected for detailed study. The various parameters affecting the measured wavelengths of minimum transmittance of the holmium oxide solution are discussed in section 3. These include such parameters as temperature, purity, concentration, and spectral bandwidth.

The spectral transmittance of the holmium oxide solution was digitally recorded on a thermal printer at 0.1 $\mathrm{nm}$ intervals with the monochromator scanning at a rate of $0.05 \mathrm{~nm} / \mathrm{s}$. The transmittance was simultaneously recorded on a chart with a wavelength display of 0.5 $\mathrm{nm} / \mathrm{cm}$.

The holmium oxide solution was contained in a 10 $\mathrm{mm}$ pathlength fused silica cuvette. The transmittance of the solution in this cuvette was measured relative to an air-only path in the reference beam.

The determination of the wavelengths of the transmittance minimum of the holmium oxide solution was derived from an analysis of the recorded digital output. The $0.1 \mathrm{~nm}$ interval transmittance data were plotted on 

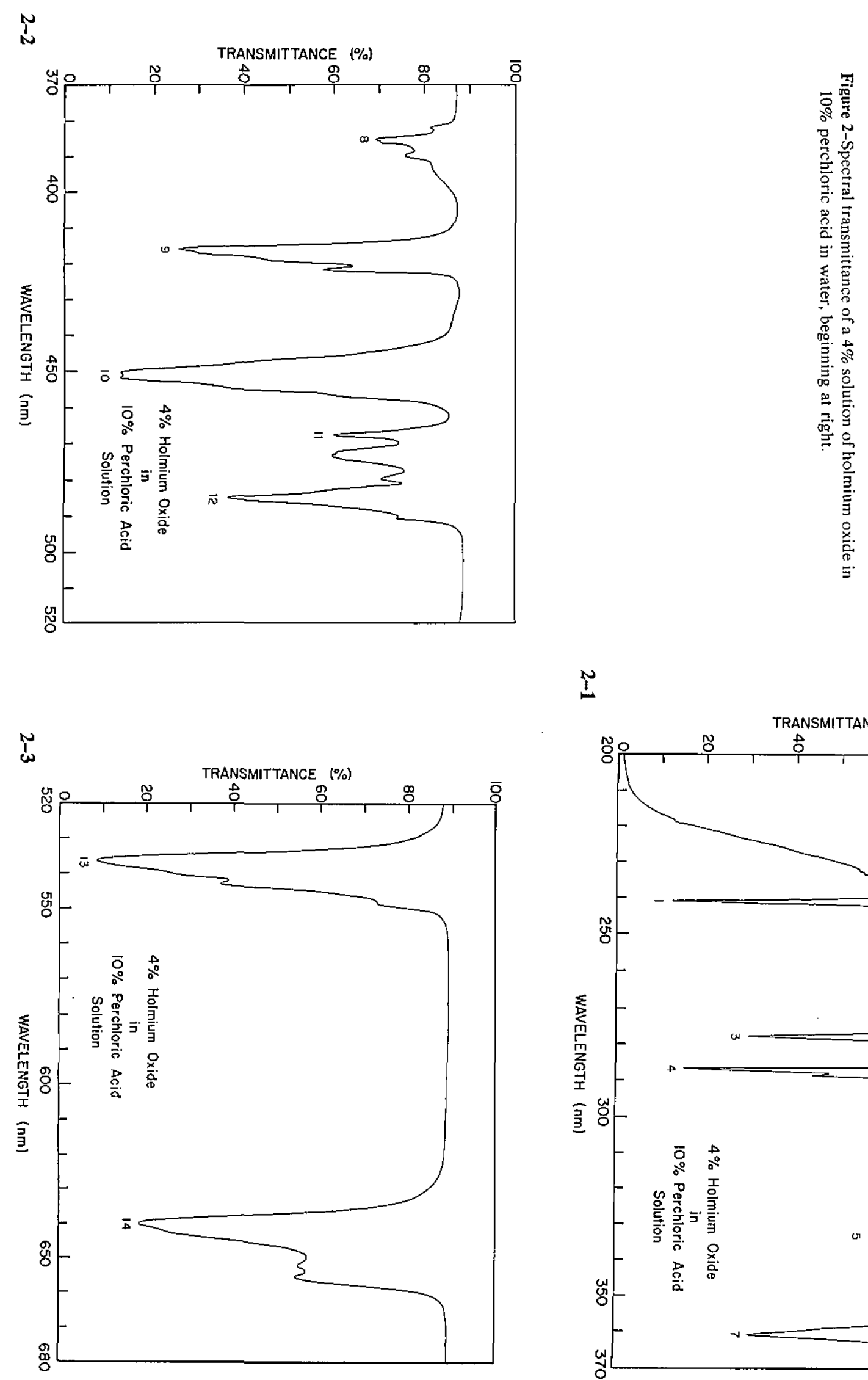

I

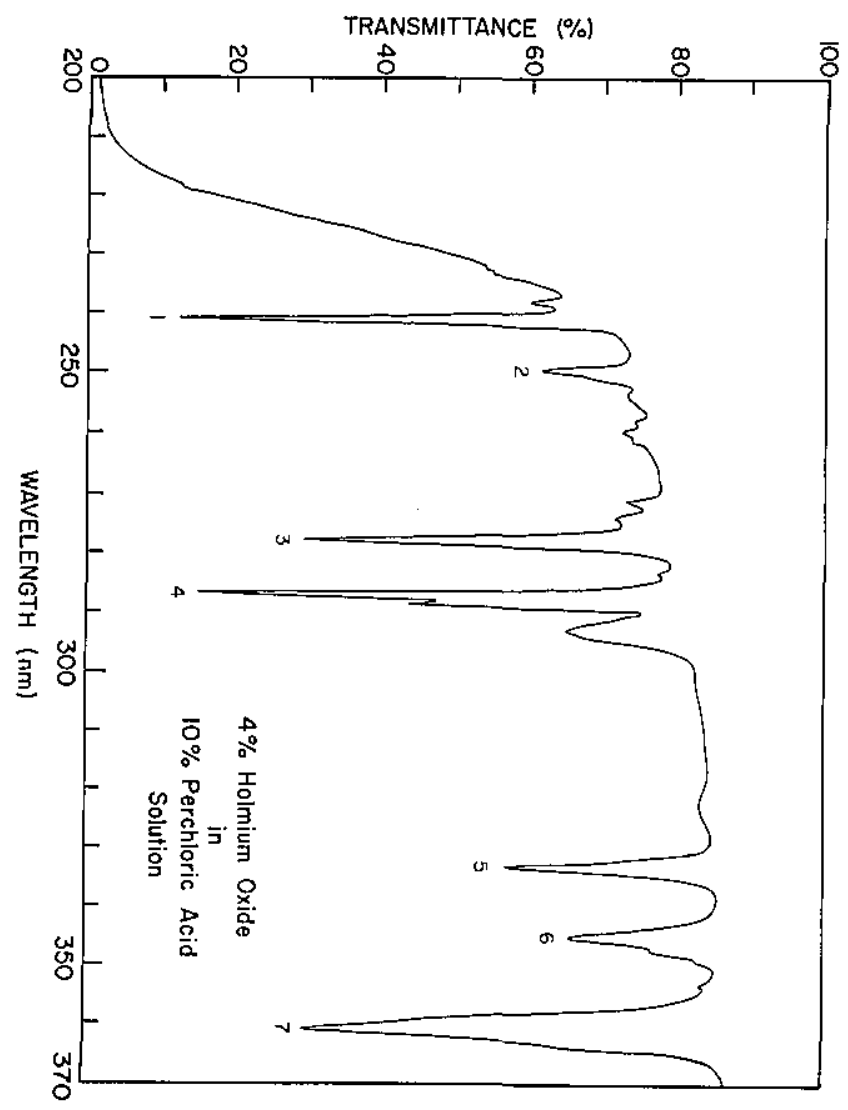


graph paper on a scale of $0.1 \mathrm{~nm}$ per $\mathrm{cm}$ with subdivisions of $0.01 \mathrm{~nm}$ per $\mathrm{mm}$ so that the wavelength interval of this plotted digital data could be read to the nearest $0.01 \mathrm{~nm}$ between the measured data points. The location of the wavelength of minimum transmittance for a given holmium oxide band was determined graphically (fig. 3) by drawing a curve through the data points and bisecting the horizontal grid lines between the two slopes of the curve representing the absorption feature. Several of these bisection points locate the line between the two slopes that intersects the minimum transmittance point, (usually at the lowest point of the curve). The wavelength at this point of intersection was taken as the measured wavelength of minimum transmittance for the absorption feature. The true wavelength of the minimum was determined by applying a wavelength correction to the instrumental wavelength scale, as determined in section 2.3.1.

\subsubsection{Accuracy of the Wavelengths of Minimum Transmittance}

The overall uncertainty in the location of the wavelengths of minimum transmittance is believed to be no greater than $\pm 0.1 \mathrm{~nm}$ at the $95 \%$ confidence limit. This conclusion is based on the reproducibility of the following calibration procedures:

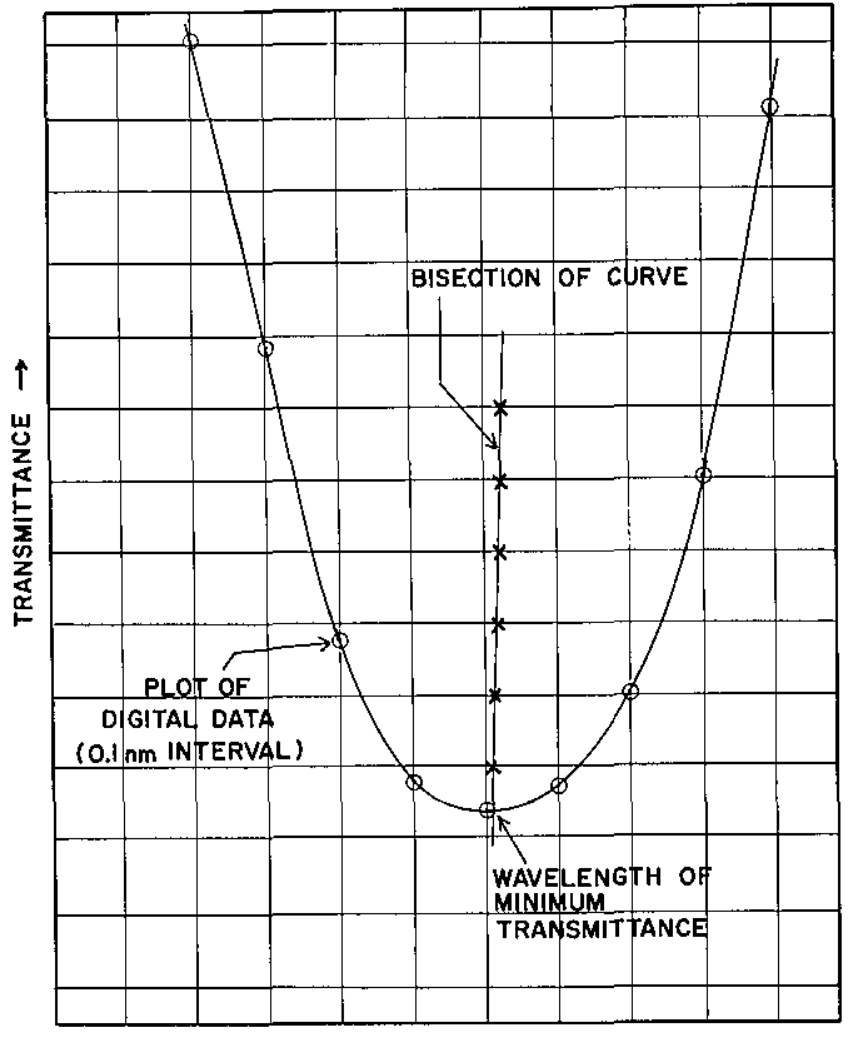

WAVELENGTH $(\mathrm{nm})$

Figure 3-Graphical technique used to determine the transmittance minima from digital spectral transmittance data.
- The uncertainty of the calibration of the instrument wavelength scale using the mercury and deuterium lamps, and the long-term and day-to-day instabilities of the instrument wavelength scale (less than $\pm 0.05 \mathrm{~nm}$ ).

- The imprecision of the graphical technique for deriving the measured wavelengths of minimum transmittance. $( \pm 0.02 \mathrm{~nm})$.

- The dependence of the measured wavelengths of minimum transmittance on variations in temperature or concentration of the solution. $( \pm 0.02 \mathrm{~nm})$.

These uncertainties have been discussed in section 2.3.1. The imprecision of the graphical technique is illustrated in table 1, where data are shown for three absorption features. Ten sets of digital results were produced by the instrument for each of these features. The digital data were plotted and the wavelengths of minimum transmittance for each absorption feature were determined for the 10 sets of data by the graphical technique. The standard deviation and standard error for the 10 determinations is also given in table 1 .

\section{Measurements}

\subsection{Influence of the Blank Cuvette, Solvent, and Water}

The spectral transmittances of an empty fused silica cuvette, a cuvette filled with distilled water, and a

Table 1. Ten cycle repetitive measurements of three holmium oxide transmittance minima. Listed minima are obtained by graphical techniques, using the digitally recorded $0.1 \mathrm{~nm}$ interval transmittance measurement.

\begin{tabular}{|c|c|c|c|}
\hline \multirow[b]{2}{*}{ Cycle } & \multicolumn{3}{|c|}{ (spectral bandwidth $=0.1 \mathrm{~nm}$ ) } \\
\hline & $\begin{array}{l}\text { Minima } \\
\text { No. } 1\end{array}$ & $\begin{array}{l}\text { Minima } \\
\text { No. } 12\end{array}$ & $\begin{array}{l}\text { Minima } \\
\text { No. } 14\end{array}$ \\
\hline 1 & $240.88 \mathrm{~nm}$ & $485.08 \mathrm{~nm}$ & $640.28 \mathrm{~nm}$ \\
\hline 2 & 240.87 & 485.10 & 640.30 \\
\hline 3 & 240.86 & 485.11 & 640.30 \\
\hline 4 & 240.84 & 485.11 & 640.30 \\
\hline 5 & 240.85 & 485.10 & 640.29 \\
\hline 6 & 240.84 & 485.11 & 640.30 \\
\hline 7 & 240.85 & 485.11 & 640.29 \\
\hline 8 & 240.85 & 485.11 & 640.30 \\
\hline 9 & 240.84 & 485.10 & 640.30 \\
\hline 10 & 240.85 & 485.11 & 640.29 \\
\hline Average: & 240.853 & 485.104 & 640.295 \\
\hline Standard & & & \\
\hline Deviation: & 0.0134 & 0.0097 & 0.0071 \\
\hline Standard & & & \\
\hline Error: & .0042 & .0031 & .0022 \\
\hline
\end{tabular}

Note: The data shown in this table have not been corrected for the wavelength scale error of the spectrophotometer. 
cuvette filled with the diluted perchloric acid (without holmium oxide) are illustrated in figure 4 . These measurements are relative to an air path in the reference beam.

Measurements of the wavelength of minimum transmittance for the band at $241 \mathrm{~nm}$ were made with the holmium oxide in perchloric acid solution versus a cuvette containing only the perchloric acid in the reference beam. The wavelength of minimum transmittance of this band was found to be the same when the holmium oxide solution was measured relative to air in the reference beam and when it was measured relative to a cuvette containing the perchloric acid solution. Since the $241 \mathrm{~nm}$ band is within the spectral range showing a slope in the transmittance of the cuvette-perchloric acid spectra, (see fig. 4) it was considered to be the band most likely to be influenced by this slope. However, no measurable influence was detected in the location of the wavelength of minimum transmittance of this band due to these spectral features associated with the solvent or cuvette.

\subsection{Influence of Temperature}

The wavelengths of minimum transmittance of the holmium oxide solution were determined at $20^{\circ} \mathrm{C}$, $25^{\circ} \mathrm{C}$, and $30^{\circ} \mathrm{C}$ for spectral bandwidths of $0.1 \mathrm{~nm}$ and $1 \mathrm{~nm}$. If there is a temperature-related influence on the location of the wavelengths of minimum transmittance, it was not detected within these temperature ranges. The measured differences were attributed to random uncertainties.

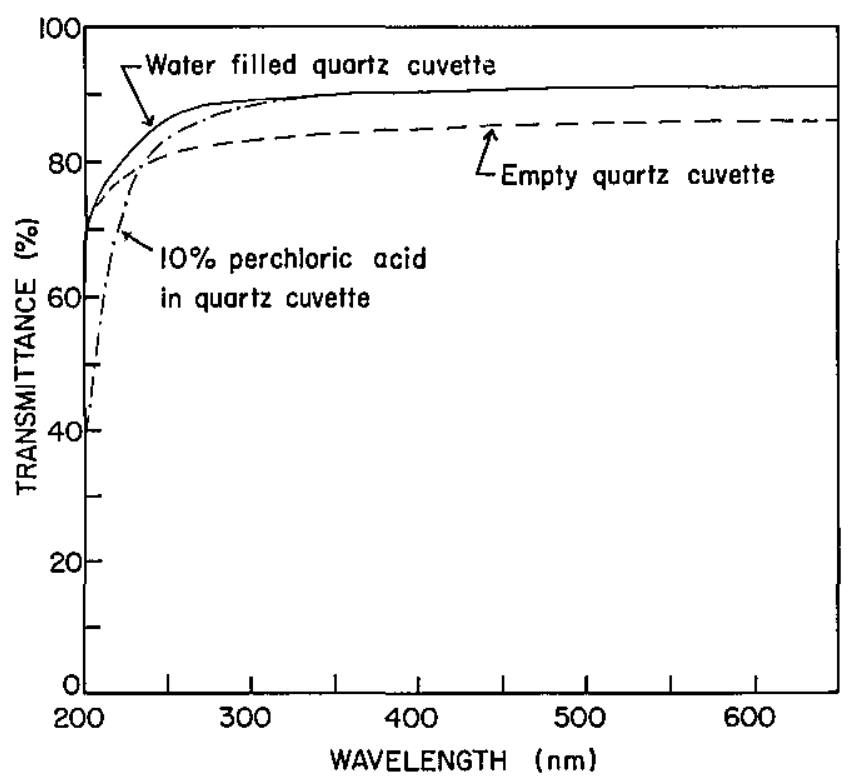

Figure 4-Spectral transmittances of an empty fused silica cuvette, a cuvette filled with water, and a cuvette filled with a solution of $10 \%$ perchloric acid in water.

\subsection{Influence of Purity}

Complete spectral scans of solutions made with the 99.99\% and $99.999 \%$ purity holmium oxides showed no spectral differences except in the extreme ultraviolet cut-off at wavelengths less than $230 \mathrm{~nm}$. The differences are illustrated in figure 5 for the wavelength range 200 to $300 \mathrm{~nm}$. The wavelengths of the minimum transmittances for the 14 selected absorption bands were found to be the same for solutions prepared from these two lots of holmium oxide.

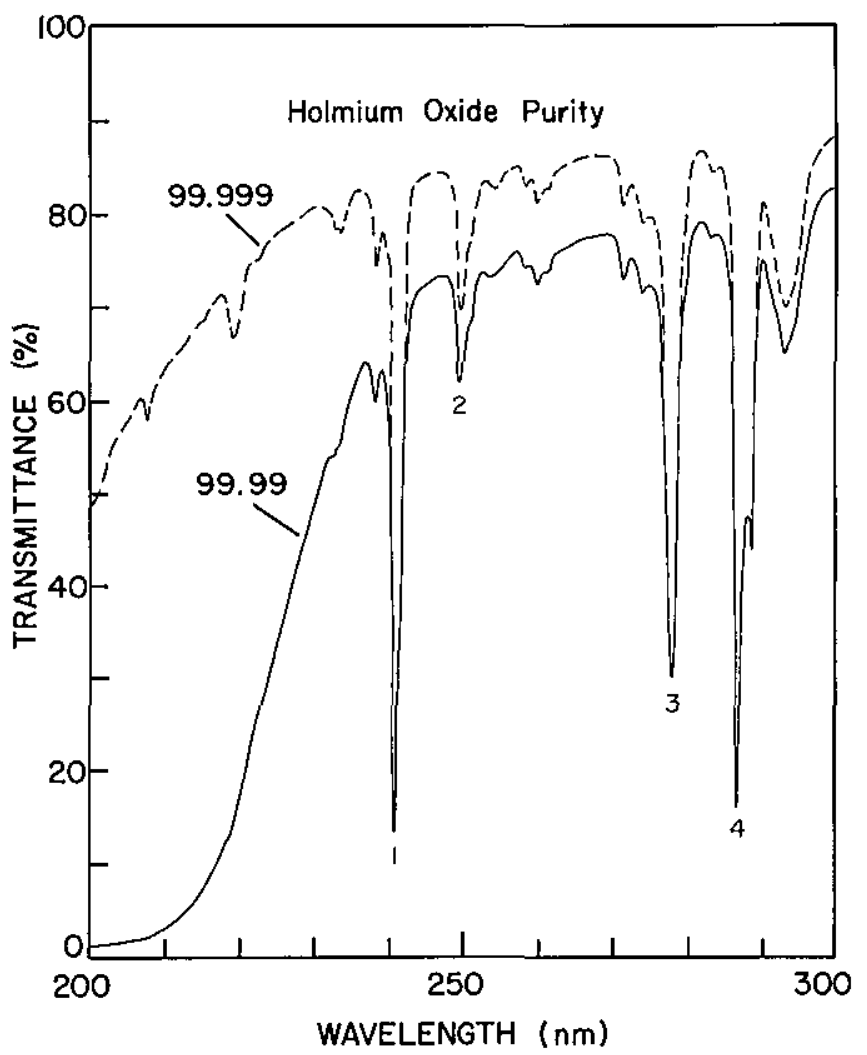

Figure 5-Spectral differences of solutions prepared from holmium oxide specimens of $99.99 \%$ purity and $99.999 \%$ purity.

\subsection{Influence of Concentration}

The location of the wavelengths of minimum transmittance of the holmium oxide solution as a function of concentration was tested with concentrations of $2 \%$, $4 \%$, and $6 \%$ in the perchloric acid solution. The general spectral scan indicated that the changes in concentration affected the measured transmittance, as would be expected but did not influence the location of the transmittance minima. To verify this further, three of the bands were evaluated by the graphical technique described in section 2.3.1. The results of these concentration measurements for these bands are shown in table 2. 
Table 2. Influence of holmium oxide concentration on the wavelengths of minimum transmittance.

\begin{tabular}{clll}
\hline \hline & \multicolumn{3}{c}{ (spectral bandwidth $=0.1 \mathrm{~nm}$ ) } \\
Holmium oxide & Minima & Minima & Minima \\
concentration & No. 1 & No. 12 & No. 14 \\
\hline $2 \%$ & $240.84 \mathrm{~nm}$ & $485.11 \mathrm{~nm}$ & $640.30 \mathrm{~nm}$ \\
$4 \%$ & 240.86 & 485.11 & 640.32 \\
$6 \%$ & 240.84 & 485.12 & 640.31 \\
\hline
\end{tabular}

Note: The data shown in this table have not been corrected for the wavelength scale error of the spectrophotometer.

\subsection{Influence of Spectral Bandwidth}

The wavelengths of minimum transmittance of the holmium oxide solution were determined for spectral bandwidths of $0.1 \mathrm{~nm}, 0.25 \mathrm{~nm}, 0.5 \mathrm{~nm}, 1 \mathrm{~nm}, 2 \mathrm{~nm}$, and $3 \mathrm{~nm}$. Measurement of the instrumental spectral bandwidths is discussed in section 2.3.2. The influence of spectral bandwidth was by far the most important parameter affecting the location of the measured wavelengths of minimum transmittance. The results of this study are shown in figures 6 through 19 for the 14 minima identified in figures $2-1,2-2$, and $2-3$. The data for bandwidth effects are also listed in tables 3 and 4 . The measurements indicate that for most of the holmium oxide bands, the location of the wavelengths of minimum transmittance does not change significantly for spectral bandwidths of less than $1 \mathrm{~nm}$. From these data it can be seen that a $0.1 \mathrm{~nm}$ spectral bandwidth is adequate to define the wavelengths of minimum transmittance within the stated uncertainties. For some bands the location of the minimum transmittance shifts only slightly for spectral bandwidths greater than $1 \mathrm{~nm}$. However, many do show large shifts for larger bandwidth settings. These results indicate that for instruments with spectral bandwidth settings of less than $1 \mathrm{~nm}$, the holmium oxide solution can serve as an excellent wavelength standard. For instruments having bandwidth settings between $1 \mathrm{~nm}$ and $3 \mathrm{~nm}$ the standard can still be of use if the instrument bandwidth is known.

\section{Results}

\subsection{Transmittance of Holmium Oxide Solution}

The general spectral signature of the holmium oxide solution is illustrated in figures $2-1,2-2$, and $2-3$ for a spectral bandwidth of $0.1 \mathrm{~nm}$. Some of the finer spectral features shown in these figures will be absent when the spectrum is recorded at bandwidths greater than $1 \mathrm{~nm}$. The major transmittance minima selected for this study are indicated by numbers 1 through 14 as shown in these figures. These band numbers are used throughout the manuscript as a key to associate the data in the tables with the spectral features illustrated in the figures.

\subsection{Selection of Useful Wavelengths of Minimum Transmittance}

The selection of holmium oxide bands that are considered useful for calibration purposes was based on the influence of spectral bandwidth on the location of these minima. The transmittance minima of small side bands associated with major absorption features usually shift in wavelength with bandwidth setting or are not resolved over the normal instrumental bandwidth range. The 14 major absorption bands are listed in tables 3 and 4. The absorption bands not listed in the tables are not considered useful for wavelength calibration purposes.

\subsection{Numerical Data}

The wavelengths of minimum transmittance of the $4 \%$ holmium oxide solution are listed in tables 3 and 4 for six spectral bandwidths. The results of the measurements as a function of temperature are also listed for $0.1 \mathrm{~nm}$ and $1 \mathrm{~nm}$ spectral bandwidths at temperature settings of $20^{\circ} \mathrm{C}, 25^{\circ} \mathrm{C}$, and $30^{\circ} \mathrm{C}$.

The shift in wavelengths of minimum transmittance as a function of spectral bandwidth is shown in figures 6 through 19 for the 14 selected minima. The recommended values of minimum transmittance are listed in table 3 and 4 for the measurements made at $25^{\circ} \mathrm{C}$.

\subsection{Uncertainty of Measured Wavelengths}

The uncertainties associated with the data listed in tables 3 and 4 have been discussed in section 2.3.4. The uncertainty in the determination of the wavelengths of minimum transmittance for the holmium oxide solution is believed to be no greater than $\pm 0.1 \mathrm{~nm}$ at the $95 \%$ confidence limit using the instrumentation and techniques described in this paper.

\subsection{Comparison of Results with Measurements Made on the NBS Reference Spectrofluorimeter}

The wavelengths of minimum transmittance of several absorption bands of holmium oxide solution were determined by analysis of spectral data obtained with the NBS Reference Spectrofluorimeter [9]. This reference instrument is primarily designed to be used as a research tool for high accuracy spectral analysis of fluorescent materials and in the development of standards for use in this area of research. The versatile design of the instrument allows for its use as a high accu- 
Figures 6-19-Fourteen holmium oxide transmittance minima as a function of spectral bandwidth.
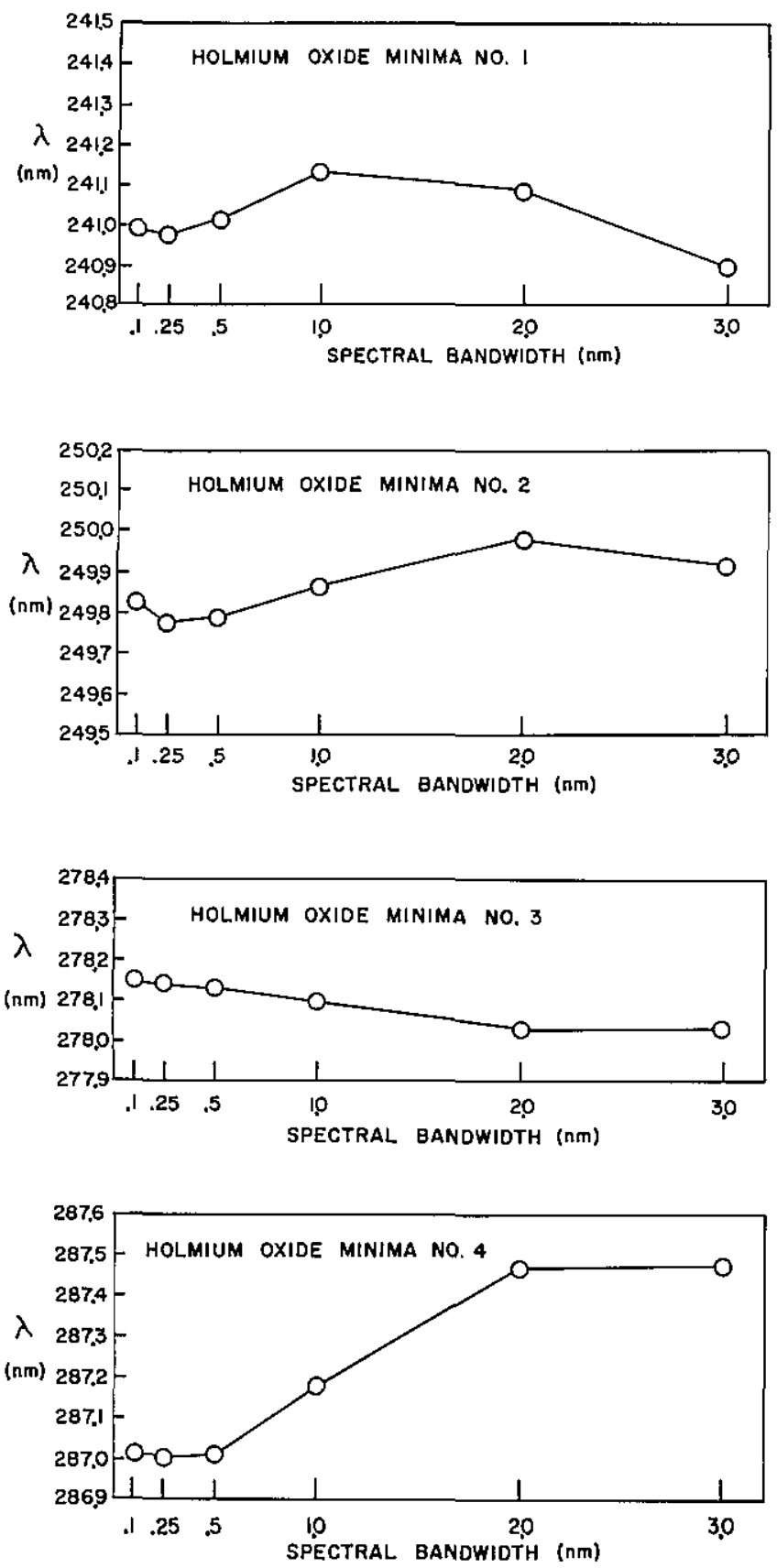
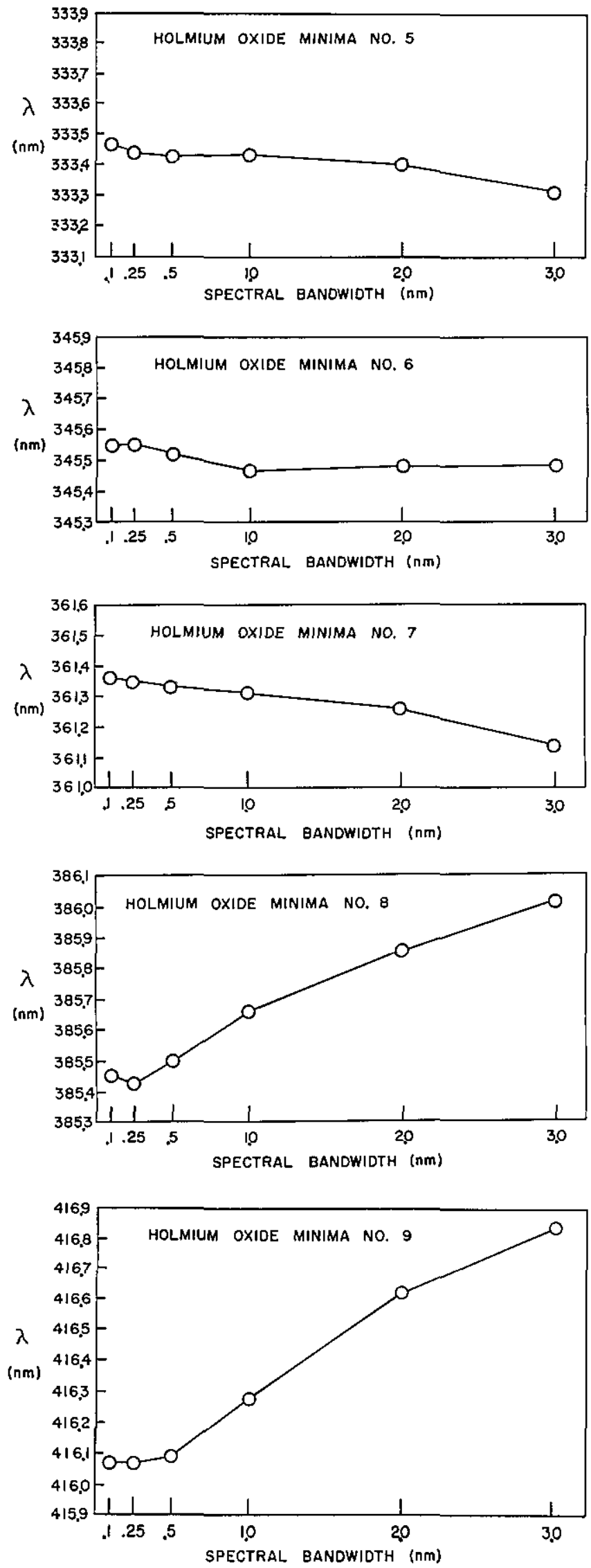

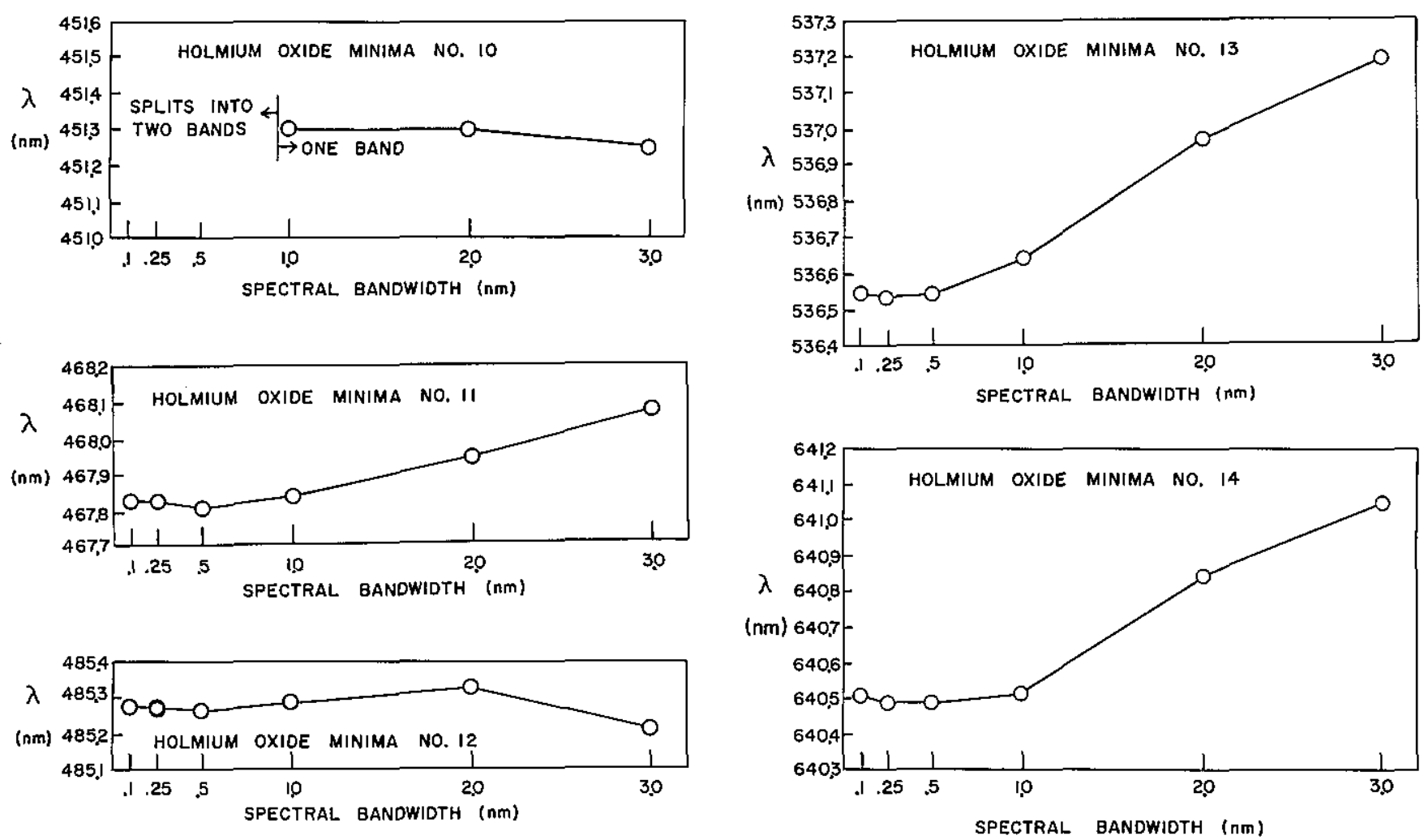

Table 3. Results of holmium oxide solution analysis for transmittance minima 1 through 7 for spectral bandwidths of $0.1,0.25,0.5,1,2$, and $3 \mathrm{~nm} .4 \%$ holmium oxide in a $10 \%$ perchloric acid solution.

\begin{tabular}{|c|c|c|c|c|c|c|c|}
\hline \multirow{2}{*}{$\begin{array}{c}\text { Minimum } \\
\text { No. }\end{array}$} & \multirow{2}{*}{$\begin{array}{l}\text { Temp. } \\
\left({ }^{\circ} \mathrm{C}\right)\end{array}$} & \multicolumn{6}{|c|}{ Spectral Bandwidths } \\
\hline & & $0.1 \mathrm{~nm}$ & $0.25 \mathrm{~nm}$ & $0.5 \mathrm{~nm}$ & $1 \mathrm{~nm}$ & $2 \mathrm{~nm}$ & $3 \mathrm{~nm}$ \\
\hline 1 & 20 & $240.99 \mathrm{~nm}$ & & & $241.13 \mathrm{~nm}$ & & \\
\hline 1 & 25 & 240.99 & $240.97 \mathrm{~nm}$ & $241.01 \mathrm{~nm}$ & 241.13 & $241.08 \mathrm{~nm}$ & $240.90 \mathrm{~nm}$ \\
\hline 1 & 30 & 240.99 & & & 241.15 & & \\
\hline 2 & 20 & 249.79 & & & 249.88 & & \\
\hline 2 & 25 & 249.83 & 249.78 & 249.79 & 249.87 & 249.98 & 249.92 \\
\hline 2 & 30 & 249.79 & & & 249.86 & & \\
\hline 3 & 20 & 278.14 & & & 278.09 & & \\
\hline 3 & 25 & 278.15 & 278.14 & 278.13 & 278.10 & 278.03 & 278.03 \\
\hline 3 & 30 & 278.14 & & & 278.09 & & \\
\hline 4 & 20 & 286.99 & & & 287.17 & & \\
\hline 4 & 25 & 287.01 & 287.00 & 287.01 & 287.18 & 287.47 & 287.47 \\
\hline 4 & 30 & 287.01 & & & 287.18 & & \\
\hline 5 & 20 & 333.48 & & & 333.45 & & \\
\hline 5 & 25 & 333.47 & 333.44 & 333.43 & 333.44 & 333.40 & 333.32 \\
\hline 5 & 30 & 333.48 & & & 333.45 & & \\
\hline 6 & 20 & 345.57 & & & 345.47 & & \\
\hline 6 & 25 & 345.55 & 345.55 & 345.52 & 345.47 & 345.49 & 345.49 \\
\hline 6 & 30 & 345.62 & & & 345.49 & & \\
\hline 7 & 20 & 361.38 & & & 361.33 & & \\
\hline 7 & 25 & 361.36 & 361.35 & 361.33 & 361.31 & 361.16 & 361.04 \\
\hline 7 & 30 & 361.38 & & & 361.31 & & \\
\hline
\end{tabular}

Note: The uncertainty in the wavelengths of minimum transmittance is $\pm 0.1 \mathrm{~nm}$. An extra decimal place is given for rounding purposes. 
Table 4. Results of holmium oxide solution analysis for transmittance minima 8 through 14 for spectral bandwidths of $0.1,0.25,0.5,1,2$, and $3 \mathrm{~nm} .4 \%$ holmium oxide in a $10 \%$ perchloric acid solution.

\begin{tabular}{|c|c|c|c|c|c|c|c|}
\hline \multirow{2}{*}{$\begin{array}{l}\text { Minimum } \\
\text { No. }\end{array}$} & \multicolumn{2}{|c|}{ Temp. } & \multicolumn{3}{|c|}{ Spectral Bandwidths } & & \\
\hline & & $\left({ }^{\circ} \mathrm{C}\right)$ & $0.1 \mathrm{nmc}$ & $\mathrm{nm} 0.5 \mathrm{~nm} 1$ & $\mathrm{~m} 3 \mathrm{~nm}$ & & \\
\hline 8 & 20 & $385.45 \mathrm{~nm}$ & & & $385.66 \mathrm{~nm}$ & & \\
\hline 8 & 25 & 385.45 & $385.42 \mathrm{~nm}$ & $385.50 \mathrm{~nm}$ & 385.66 & $385.86 \mathrm{~nm}$ & $386.01 \mathrm{~nm}$ \\
\hline 8 & 30 & 385.44 & & & 385.68 & & \\
\hline 9 & 20 & 416.10 & & & 416.27 & & \\
\hline 9 & 25 & 416.07 & 416.07 & 416.09 & 416.28 & 416.62 & 416.84 \\
\hline 9 & 30 & 416.13 & & & 416.30 & & \\
\hline 10 & 20 & ----_* & & & 451.30 & & \\
\hline 10 & 25 & -.--* & -_-_** & - & 451.30 & $4 \$ 1.30$ & 451.24 \\
\hline 10 & 30 & ----_* & & & 451.34 & & \\
\hline 11 & 20 & 467.83 & & & 467.84 & & \\
\hline 11 & 25 & 467.82 & 467.82 & 467.80 & 467.83 & 467.94 & 468.07 \\
\hline 11 & 30 & 467.84 & & & 467.84 & & \\
\hline 12 & 20 & 485.30 & & & 485.29 & & \\
\hline 12 & 25 & 485.28 & 485.28 & 485.27 & 485.29 & 485.33 & 485.21 \\
\hline 12 & 30 & 485.31 & & & 485.30 & & \\
\hline 13 & 20 & 536.53 & & & 536.65 & & \\
\hline 13 & 25 & 536.54 & 536.53 & 536.54 & 536.64 & 536.97 & 537.19 \\
\hline 13 & 30 & 536.45 & & & 536.63 & & \\
\hline 14 & 20 & 640.48 & & & 640.52 & & \\
\hline 14 & 25 & 640.51 & 640.49 & 640.49 & 640.52 & 640.84 & 641.05 \\
\hline 14 & 30 & 640.52 & & & 640.49 & & \\
\hline
\end{tabular}

* Splits into 2 minima for spectral bandwidths less than $1 \mathrm{~nm}$

Note: The uncertainty in the wavelengths of minimum transmittance is $\pm 0.1 \mathrm{~mm}$. An extra decimal place is given for rounding purposes.

racy spectrophotometer in some applications. The wavelength scale of the spectrofluorimeter has been carefully calibrated by extensive measurements of emission line sources and is known to have an uncertainty of $\pm 0.1 \mathrm{~nm}$ for spectral bandwidth of $0.1 \mathrm{~nm}$.

This reference instrument was used to confirm the results obtained with the calibrated high-precision commercial spectrophotometer used for the holmium oxide measurements. A comparison was made to confirm the results for three of the transmittance minima at one spectral bandwidth setting. The results of this comparison are shown in table 5 . The two instruments provided data for these three transmittance minima that agree to within $0.1 \mathrm{~nm}$. The wavelength scale uncertainty for both instruments is $\pm 0.1 \mathrm{~nm}$.

\subsection{Other Measurements Outside NBS}

A list of the wavelengths of minimum transmittance of the holmium oxide solution reported by other workers is given in table 6. (Ref. 10 offers details of these measurements.)
Table 5. Comparison of values for holmium oxide transmittance minima with values obtained with the NBS Reference Spectrofluorimeter.

\begin{tabular}{llll}
\hline \hline & \multicolumn{3}{c}{ (spectral bandwidth $=0.1 \mathrm{~nm}$ ) } \\
& $\begin{array}{l}\text { Minima } \\
\text { No. 1 }\end{array}$ & No. 7 & $\begin{array}{l}\text { Minima } \\
\text { No. 14 }\end{array}$ \\
\hline $\begin{array}{l}\text { Varian (Cary) } \\
\text { Model 2390 }\end{array}$ & $240.995 \mathrm{~nm}$ & $361.361 \mathrm{~nm}$ & $640.507 \mathrm{~nm}$ \\
$\begin{array}{l}\text { NBS Reference } \\
\text { Spectrofluorimeter }\end{array}$ & $240.970 \mathrm{~nm}$ & $361.313 \mathrm{~nm}$ & $640.469 \mathrm{~nm}$ \\
\hline
\end{tabular}

The data shown in table 6 indicate that workers in other laboratories are in generally good agreement with one another and that the NBS data also agree well with these workers' previously published data. These workers also found that the wavelengths of minimum transmittance of holmium oxide in similar solution in perchloric acid are not sensitive to variations in temperature and concentration. They also concluded that the wavelengths of minimum transmittance were least affected by changes in spectral bandwidth for band- 
Table 6. Comparison of values for holmium oxide transmittance minima with values obtained by other workers (see Reference 11).

\begin{tabular}{|c|c|c|c|c|c|c|}
\hline $\begin{array}{l}\text { Min. } \\
\text { No. }\end{array}$ & $\begin{array}{c}\text { NBS } \\
\text { Transmittan } \\
\text { Minima } \\
\end{array}$ & nce & (1) & $(2)$ & $(3)$ & (4) \\
\hline 1 & $240.99 \mathrm{~nm}$ & & $241.15 \mathrm{~nm}$ & $241.0 \mathrm{~nm}$ & $241.1 \mathrm{~nm}$ & $241.1 \mathrm{~nm}$ \\
\hline 2 & 249.83 & * & 249.75 & 250.0 & 249.7 & 249.7 \\
\hline 3 & 278.15 & * & 278.2 & 277.8 & 278.7 & 278.2 \\
\hline 4 & 287.01 & * & 287.15 & 287.5 & 287.1 & 287.2 \\
\hline 5 & 333.47 & * & 333.5 & 333.3 & 333.4 & 333.3 \\
\hline 6 & 345.55 & * & 345.6 & 345.5 & 345.5 & 345.0 \\
\hline 7 & 361.36 & & 361.5 & 361.0 & 361.5 & 361.2 \\
\hline 8 & 385.45 & $*$ & 385.6 & 385.6 & 385.5 & 385.6 \\
\hline 9 & 416.07 & $*$ & 416.2 & 416.0 & 416.3 & 416.6 \\
\hline 10 & 451.30 & $\#$ & 450.7 & 450.4 & 450.8 & 451.0 \\
\hline 11 & 467.82 & & 467.75 & & & 468.0 \\
\hline 12 & 485.28 & & 485.25 & 485.2 & 485.8 & 485.2 \\
\hline 13 & 536.54 & & 536.3 & & & 536.8 \\
\hline 14 & 640.51 & & 640.5 & & & \\
\hline
\end{tabular}

* Spectral bandwidth $=0.1 \mathrm{~nm}$

\# Spectral bandwidth $=1 \mathrm{~nm}$

widths less than $1 \mathrm{~nm}$, but that large shifts can be encountered at bandwidths in excess of $1 \mathrm{~nm}$.

\section{Conclusions}

The reported wavelengths of minimum transmittance of the holmium oxide solution appearing in tables 3 and 4 are estimated to be uncertain by no more than \pm 0.1 $\mathrm{nm}$ at the $95 \%$ confidence limit. These wavelengths of minimum transmittance were found to be essentially unaffected by changes in temperature at $25^{\circ} \mathrm{C} \pm 5^{\circ} \mathrm{C}$. They were also unaffected by variations in the concentration for solutions containing $2 \%, 4 \%$, and $6 \%$ holmium oxide. The critical parameter affecting the measured values of minimum transmittance was found to be the spectral bandwidth setting of the spectrophotometer. For spectral bandwidths less than $1 \mathrm{~nm}$ the wavelength shift is generally less than $0.2 \mathrm{~nm}$. Users can most effectively determine the wavelength error associated with their instrument by using the NBS data listed in tables 3 and 4 that are representive of the spec tral bandwidth setting ordinarily used with the instru ment. The wavelengths of minimum transmittance of the holmium oxide solution for spectral bandwidths greater than $3 \mathrm{~nm}$ have not been evaluated.

The authors gratefully acknowledge the assistance of Chenq-Tsong Chang, guest worker from Taiwan, for his contribution in measuring the transmittance minima of the holmium oxide solution on the NBS Reference Spectrofluorimeter; Jack J. Hsia, for constructive discussions; Robert W. Burke, for assistance in the preparation of various $\mathrm{Ho}_{2} \mathrm{O}_{3}$ solutions; and the NBS Optical Shop for preparation of the fused silica cuvettes.

\section{References}

[1] The terminology used in this work was defined earlier by K. D. Mielenz in Anal. Chem. 48, 1093 (1976).

[2] Keegan, H. J.; J. C. Schleter and V. R. Weidner, Ultraviolet Wavelength Standard for Spectrophotometry, J. Opt. Soc. Am. 51, 1470 (1961), (abstract).

[3] Venable, W. H., Jr., and K. L. Eckerle, Standard Reference Material: Didymium Glass Filters for Calibrating the Wavelength Scale of Spectrophotometers (SRM 2009, 2010, 2013, 2014).

[4] The holmium oxide was obtained from Research Chemicals, a Division of Nucor Corp., Phoenix, Arizona.

[5] Gschneider, K. A., Jr., and L. E. Yring, Handbook of the Physics and Chemistry of Rare Earths, Vol. 3 North-Holland (1979).

[6] Harrison, G. R., MIT Wavelength Tables, MIT Press, Cambridge, MA (1960).

[7] ASTM Manual on Recommended Practices in Spectrophotometry, Published by American Society for Testing and Materials, 1916 Race St., Philadelphia, PA 19103 (1969).

[8] CRC Handbook of Chemistry and Physics, 63d Edition, CRC Press, Inc., (1982-1983).

[9] Mielenz, K. D., in "Measurement of Photoluminescence" (K. D. Mielenz, Editor), pp 17-19, Academic Press (1982).

[10] Burgess, C., and A. Knowles, Eds. Standards in Absorption Spectrometry, Chapter 7, Wavelength Calibration, Chapman and Hall, London (1981). 